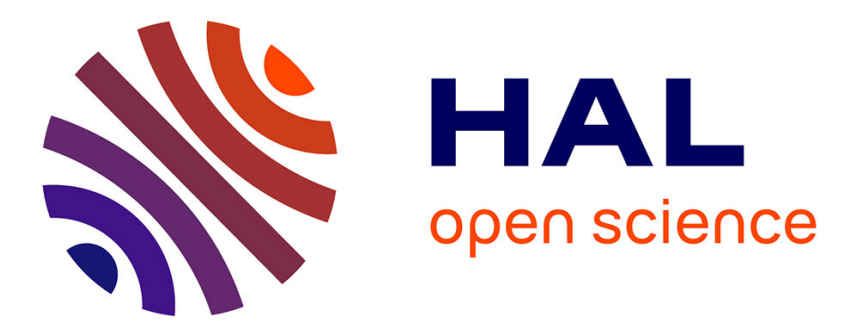

\title{
Flow velocity estimation using ultrasound and wavelet transformation
}

Cheick Guetbi, Denis Kouamé, Abdeldjalil Ouahabi, Jean-Paul Chemla, L. Bensaada

\section{- To cite this version:}

Cheick Guetbi, Denis Kouamé, Abdeldjalil Ouahabi, Jean-Paul Chemla, L. Bensaada. Flow velocity estimation using ultrasound and wavelet transformation. 24th Annual Conference of the IEEE Industrial Electronics Society (IECON 1998), Aug 1998, Aachen, Germany. pp.1366-1369, 10.1109/IECON.1998.722850 . hal-03153368

\section{HAL Id: hal-03153368 https://hal.science/hal-03153368}

Submitted on 1 Mar 2021

HAL is a multi-disciplinary open access archive for the deposit and dissemination of scientific research documents, whether they are published or not. The documents may come from teaching and research institutions in France or abroad, or from public or private research centers.
L'archive ouverte pluridisciplinaire HAL, est destinée au dépôt et à la diffusion de documents scientifiques de niveau recherche, publiés ou non, émanant des établissements d'enseignement et de recherche français ou étrangers, des laboratoires publics ou privés. 


\section{FLOW VELOCITY ESTIMATION USING ULTRASOUND SIGNALS AND WAVELET TRANSFORMATION}

\author{
C. Guetbi, D. Kouamé, \\ A. Ouahabi and J.P. Chemla \\ LUSSI/GIP Ultasons, E.I.T. \\ 7 Avenue Marcel Dassault BP 407 \\ 37204 Tours Cedex 3, France \\ Tel : (02) 47711226 - Fax: (02) 47279533 \\ guetbi@univ-tours.fr
}

\author{
L. Bensaada \\ Institut des télécommunications \\ Oran, Algérie \\ bensaada@hotmail.com
}

\begin{abstract}
It is well known that classical time delay estimation gives an inexact estimation of the true delay between two signals delayed by non-integral multiple of the sample period. For more accurate estimation, new methods are proposed to estimate small time delay with regard to sample period of ultrasonic signals. In this paper, we focus on two methods based on wavelet transform for time delay estimation between ultrasound emitted pulse and received signal. The first technique consists in computing at a fixed frequency (which is the emitting frequency of the ultrasonic signal) the difference of maximum time argument between wavelet coefficients of the two signals (the reference and the delayed signal). The second technique, based on a crosswavelet representation, directly provides the time delay between the two signals. The performance of these methods in terms of bias and variance are compared to classical correlation using polynomial interpolation. Numerical results show the superior performance of the crosswavelet approach for time delay estimation.
\end{abstract}

\section{INTRODUCTION}

Accurate determination of absolute velocities has been often hindered by unsatisfactory time measurements, due to small delays in the order of fraction of the sample interval. Consequently, non-integral multiple of sample period time delay estimation is an important issue in ultrasound flow velocimetry. The usual methods for this purpose are derived from classical technique widely described in signal processing literature [1], [2]. These techniques consist of identifying the maximum value of the cross correlation between the reference and the delayed signal, and then applying an interpolation function to extract delay. The effects of using sampled signals and of interpolating to perform analog processing of the time delay parameter are studied in [3]. In this paper, we provide an alternative to classical correlation methods by the use of wavelet transformation.

Two methods are then described:

The first one consists in computing the wavelet coefficients of each signal with a fixed compression/dilatation factor (this factor corresponds to the central frequency of the emitting signal). The time delay is obtained from the peak coordinate of these two wavelet coefficients. The second method is based on crosswavelet, which consists in combining the two wavelet transforms of emitted and received signal [4]. The peak of this wavelet transform directly provides the time delay. These methods have been evaluated on simulated ultrasonic signals and their performance, in terms of bias and variance, are compared to the conventional cross correlation method. This work is restricted to ultrasonic signals in high SNR situation. The paper is organized as follows. In Section II, the measurement principle of flow velocity estimation is presented. In Section III, we introduce two processing methods based on wavelets. In Section IV, the performance of the estimators is characterized using numerical results. Finally, we draw our conclusions in Section V.

\section{EXPERIMENTAL CONDITIONS}

One of the main purposes of ultrasound flow velocimetry is the realization of liquid or gaz flow meter. Two principles are used for this aim. The first one is the Doppler technique: flowing fluid is insonified with an ultrasound emitted at frequency $f_{0}$. This fluid bakscatters the ultrasound at frequency $f_{0}+f_{d}$, where $f_{d}$ is proportional to the fluid velocity. Therefore, this technique depends on the choice of the emitting signal frequency and assumes that the output signal is a narrowband model [5]. Although the narrowband model is generally valid for radar applications since the celerity $\mathrm{c}$ is quite large, it is not always valid in acoustic environments. The second technique, which will be considered here, is based on the measurement of the time delay between two ultrasound signals which have crossed a flowing fluid, after their emission (see Fig. 1).

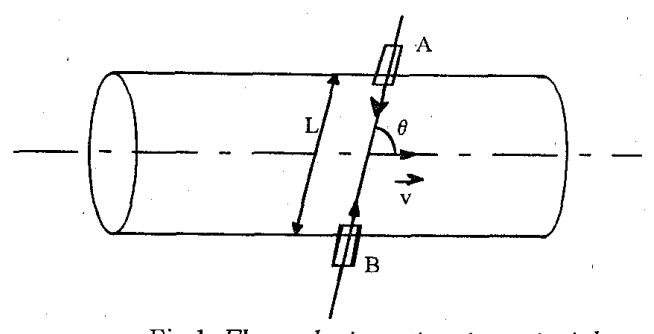

Fig.1. Flow velocity estimation principle 
Two piezoelectric transducers $A$ and $B$ are placed with an angle $\theta$ with respect to the flowing direction, across a pipe containing a flowing fluid. After having been simultaneously excited, the ultrasound signals received by the two transducers are delayed by a time, which is proportional to the velocity $v$ of the fluid.

If $\tau_{A B}$ is the time flight from A to B (Fig.1) and $\tau_{B A}$ from $B$ to $A$, it can be shown that

$$
\tau_{A B}=\frac{L}{c+v \cos \theta}
$$

Where $L$ is the distance between the two transducers and $c$ the ultrasound celerity in the fluid. The velocity is then given by

$$
v=\frac{L}{2 \cos \theta} \frac{\tau_{A B}-\tau_{B A}}{\tau_{A B} \tau_{B A}}
$$

One of the advantages of this technique is that the narrowband assumption is no more necessary.

Here, in order to take into account slow flows, we have to measure non multiple of sampling period time delay. The main problem encountered by the classical techniques such as 1-D correlation processing is the large errors yielded in these conditions [6]. In the following section, we will emphasis on two new techniques for subsample time delay measurement in the context of the velocimetry.

\section{PROCESSING METHODS}

The following model of signals received by two sensors represents the time delay estimation (T.D.E) problem:

$$
\begin{gathered}
x(t)=s(t)+n_{1}(t) \\
y(t)=A s\left(t-\tau_{0}\right)+n_{2}(t),
\end{gathered}
$$

Where $n_{1}(t)$ and $n_{2}(t)$ are two measurements noises uncorrelated with each other and with the ultrasonic signal $s(t)$. The problem is to estimate $\tau_{0}$ using a finite set of samples of $x(t)$ and $y(t)$.

\section{A. T.D.E using wavelet representation with fixed scale}

The wavelet transform of a signal $x(t)$, defined as the correlation between the signal and a set of basic wavelets $h_{s, t}(t)$, is expressed as

$$
C_{x}(s, \tau)=\int_{-\infty}^{+\infty} x(t) h_{s, \tau}^{*}(t) d t
$$

Where $h(t)$, referred to as the mother wavelet, must satisfy the admissibility conditions and * denotes the complex conjugate. The wavelet transformation is applied to both emitted and received signal. When the signal is detected by one of the matched filter $h_{s . t}(t)$, there is a correlation peak, whose coordinates indicate the dilation factor $s$ and the position $\tau$ of the signal. Let $x(t)$ and $y(t)$ be the signal received by the two transducers, and $C_{x}(\tau)$ and $C_{y}(\tau)$ their wavelet transforms at a fixed scale $s_{0}$ :

$$
\begin{aligned}
& C_{x}(\tau)=\int x(t) \psi\left(\frac{t-\tau}{s_{0}}\right) d t \\
& C_{y}(\tau)=\int y(t) \psi\left(\frac{t-\tau}{s_{0}}\right) d t
\end{aligned}
$$

Where $s_{0}=5 / 2 \pi f_{0}$ and $f_{0}$ is the central frequency of $x(t)$. The time delay $\tau_{0}$ between $x(t)$ and $y(t)$ is given by

$$
\tau_{0}=\operatorname{Arg} \max \left[C_{x}(\tau)-C_{y}(\tau)\right]
$$

The mother wavelets that can represent the filtered response of the transducer are gaussian signal modulated as Morlet wavelet or Mexican hat because they have a shape similar to the ultrasonic signal.

The chosen mother wavelet for this method is the Morlet wavelet which real part is plotted in Fig. 2 .

$$
\psi(t)=e^{-\left(t^{2} / 2\right)} e^{i 5 t}
$$

With this method the accurate time delay can be given without interpolation. The resolution is then defined by the time variable $\tau$.

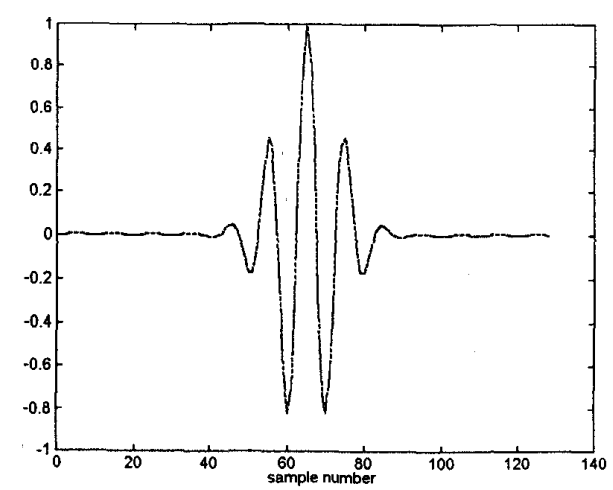

Fig.2. Morlet mother wavelet.

\section{B. Crosswavelet transformation}

As defined above, the direct wavelet transform has a high qualitative interest but it needs to be extended to 
make it robust for accurate time and/or frequency measurements.

One of the main limitations of the common velocity estimation methods is that they have good behavior mainly with narrowband signals.

The narrowband assumption for a ultrasound signal in a flowing fluid is expressed as $2 v / c<<1 / T B$, where $T$ is the signal duration and $B$ its bandwidth. This assumption of narrowband processing can be violated either if the velocity of the scatters is high or if the time-bandwidth product $T B$ is large. When the narrowband assumption is no more valid, the conventional methods are highly biased [7]. In the wideband case, a time-delayed and scaled replica of the source signal approximates the received signal:

$$
y(t)=\frac{1}{\sqrt{\left|s_{0}\right|}} x\left(\frac{t-\tau_{0}}{s_{0}}\right)
$$

Where $1 / \sqrt{s}_{0} \mid$ is energy normalization.

Assume that $x(t)$ is the transmitted pulse and $y(t)$ is the received signal and calculate the wavelet transform of $y(t)$ with respect to $x(t)$

$$
C_{x y}(s, \tau)=\frac{1}{\sqrt{s}} \int_{-\infty}^{+\infty} x(t) y^{*}\left(\frac{t-\tau}{s}\right) d t
$$

The highest correlation provides the scaling factor and the time delay between $x$ and $y$

$$
\left(s_{\max }, \tau_{\max }\right)=\operatorname{Arg} \max \left[C_{x y}(s, \tau)\right]
$$

When the emitted signal is unknown analytically (i.e only a digitized version is available), then creating replicas may be computationally intensive.

To overcome these difficulties, the processing is performed in the wavelet transform domain and relies on the cross wavelet transform [8].

$C_{x y}(s, \tau) \propto \iint C_{\psi x}(a, b) \cdot C_{\psi y}^{*}\left(\frac{a}{s}, \frac{b-\tau}{s}\right) \frac{d a d b}{a^{2}}$

where $C_{\psi x}$ is the wavelet transform of $x(t)$.

Since the replicas $\psi\left(\frac{t-b}{a}\right)$ with respect to the mother wavelet $\psi(\mathrm{t})$ can be computed in advance, the wavelet transforms will be available quickly.

\section{PERFORMANCE EVALUATION}

We examine two time delay values which are nonintegral multiple of the sample period Ts $\tau_{1}=0.66 \times T_{s}$ and $\tau_{2}=1.32 \times T_{s}$. The emitted ultrasonic pulse used in our simulation is modeled by

$$
x(t)=\left(\frac{t}{3 \alpha}\right)^{3} \sin \left(2 \pi f_{0} t\right) e^{\left(3-\frac{t}{\alpha}\right)}
$$

with center frequency $f_{0}=2 \mathrm{MHz}$ and sampling frequency $F_{S}=1 / T_{s}=20 \mathrm{MHz}$. The ultrasonic simulated pulse corrupted with additive noise is shown in Fig.3. The model of signals received by two sensors is defined in (3).

As it is seen in Fig.2, the Morlet wavelet is a suitable candidate to be an analyzing wavelet.

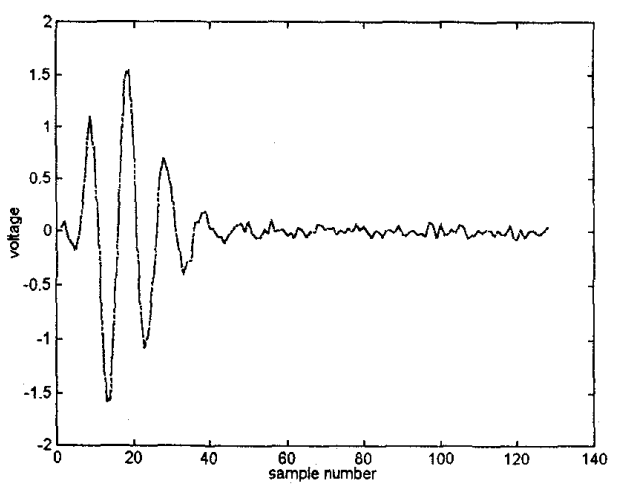

Fig.3. Emitted ultrasonic signal corrupted by noise.

In order to quantify the improvement of time delay estimation for the two methods, the estimated time delay between emitted and received signals are evaluated as a function of signal-to-noise ratio, therefore, the bias and standard deviation of the estimators are performed on 50 realizations.

\section{A. Illustrative examples}

We evaluate the estimators with two time delay values $\tau_{1}=0.66 \times T_{s}$ and $\tau_{2}=1.32 \times T_{s}$. These values are nonintegral multiple of the sample period $T_{s}$.

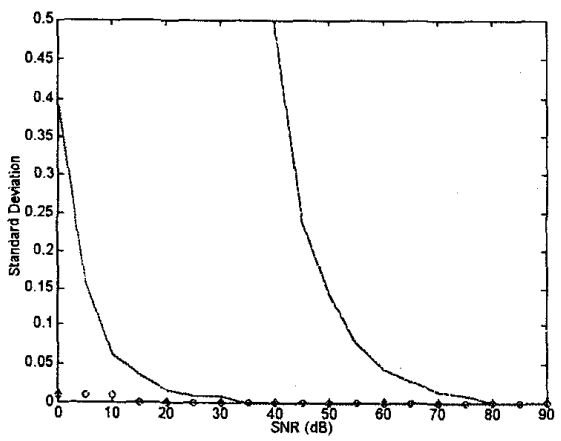

Fig.4a. Standard deviation vs. SNR for TDE: $\tau=2.7 I \times T_{s}$ 


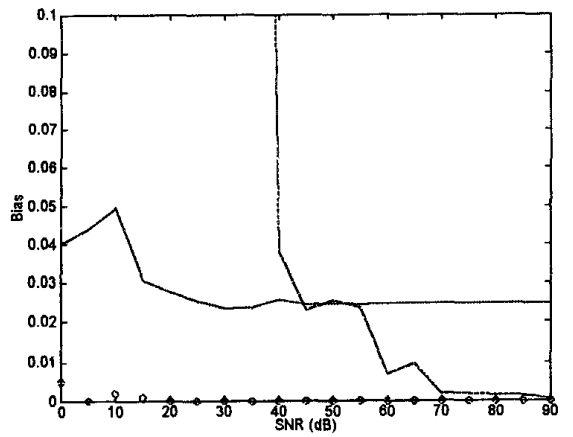

Fig.4b. Bias vs. SNR for TDE; $\tau=2.71 \times T_{s}$

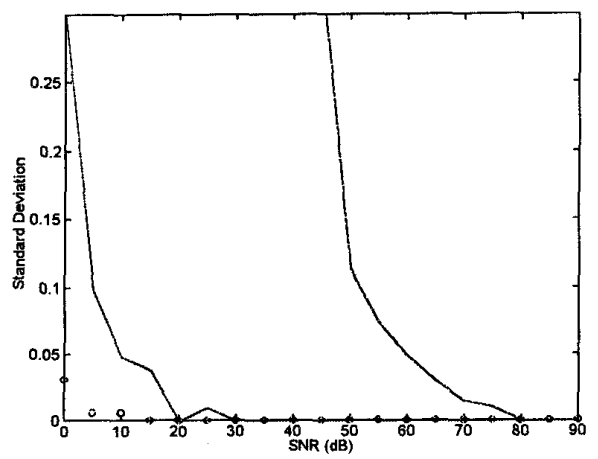

Fig.5a. Standard deviation vs. SNR for TDE; $\tau=0.66 \times T_{s}$

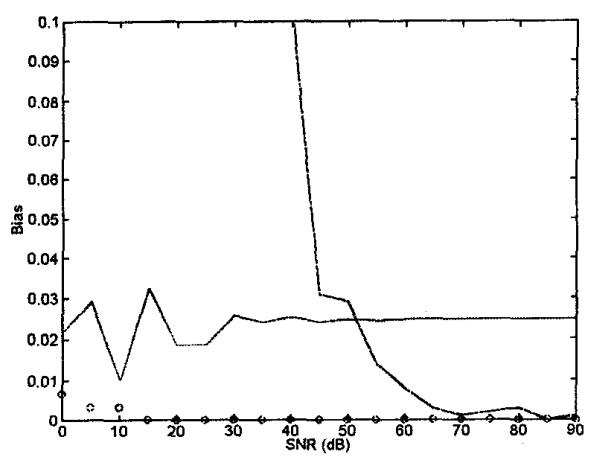

Fig.5b. Bias vs. SNR for TDE; $\tau=0.66 \times T_{s}$

o: Crosswavelet approach

- Wavelet transform

---: Cross-correlation with polynomial interpolation

\section{B. Discussion}

The figure (4) and (5) exhibit the performances of time delay estimators in terms of bias and standard deviation $v s$. signal-to-noise ratio. It is clear that the crosswavelet approach is hardly unbiased. This approach has a better behavior than with the classical correlation both from computational and accuracy viewpoints, because it is well adapted in wideband ultrasonic signals. Moreover, wavelets can cover a continuous range of time delay values with fine resolution.

Note that T.D.E based on wavelets at a fixed scale presents a better performance than T.D.E with classical correlation using interpolation only for very high SNR, but they have an equivalent computational complexity. Although, replicas with respect to the mother wavelet can be computed in advance, thus computational complexity for T.D.E with crosswavelet tool is quite high.

\section{CONCLUSION}

Two techniques using wavelet transform for improving time delay estimation of ultrasonic echoes were presented and compared with correlation method using polynomial interpolation.

Numerical results show that crosswavelet is hardly unbiasedand. Consequently, it is well adapted to estimate time delay and then flow velocity since the Morlet wavelet is suitable to analyze an ultrasonic pulse. These preliminary results are very encouraging step for studying more complex flows.

\section{REFERENCES}

[1] I. Céspedes, Y. Huang, J. Ophir and S. Spratt, "Methods for Estimation of Subsample Time Delays of Digitized Echo Signals", Ultrasonic Imaging, vol.17, pp. 142-171, 1995.

[2] Charles H. Knapp and G. Clifford Carter, "The Generalized Correlation Method for Estimation of Time Delay", IEEE Trans.ASSP, vol. ASSP-24, pp. 320-327, 1976.

[3] Giovanni Jacovetti and Gaetano Scarano, "Discrete Time Technique for Time Delay Estimation", IEEE Trans SP, vol. 41, n², pp. 525-533, 1993

[4] R. K. Young, "Wavelet Theory and its Applications, Kluwer Academic Publishers", Boston, 1993.

[5] L. G. Weiss, "Time-Varying system Characterization for Wideband Input Signal", Signal Processing, vol. 55, pp.295-304, 1996.

[6] J. P. Ianniello, "Time Delay Estimation via Cross-Correlation in the Presence of Large Estimation Errors", IEEE Trans. ASSP, vol. ASSP-30, n.6, pp. 998-1003, 1982.

[7] O. Bonnefous and P. Pesque "Time Domain Formulation of Pulse-Doppler Ultrasound and Blood Velocity Estimation by Cross-Correlation", Ultrasonic Imaging, vol. 8, pp. 73-85, 1986. [8] L. G. Weiss, "Wavelets and Wideband Correlation processing", IEEE Signal Processing Magazine, pp. 13-32, 1994. 